\title{
Future challenges of spinal cord infarction treatment
}

\author{
Dominika Jakubowicz-Lachowska, Joanna Tarasiuk, Katarzyna Kapica-Topczewska, \\ Anna Mirończuk, Jan Kochanowicz, Alina Kułakowska \\ Department of Neurology, Medical University of Bialystok, Bialystok, Poland
}

We greatly appreciated and were impressed by the review article by Wyszomirski et al. looking at the evidence regarding the efficacy of intra-venous (IV) and intra-arterial (IA) thrombolysis with recombinant tissue plasminogen activator (rt-PA) and mechanical thrombectomy (MT) in the treatment of acute basilar artery occlusion (BAO) [1], and Lattanzi et al. who reviewed the state of current knowledge about future challenges of stroke management [2], as well as the response to the letter 'Future challenges of stroke treatment' written by Karaszewski et al. [3]. To date, only a few studies concerning BAO treatment with rt-PA have been published, but with no randomised clinical trials [1]. The treatment of acute BAO is still based upon consensus, clinical practice, and estimations of clinical benefits [2].

In relation to the articles written by Wyszomirski et al., Lattanzi et al. and Karaszewski et al., we would like to emphasise that a similar problem concerns the treatment of spinal cord infarction (SCI), which accounts for $0.3-1 \%$ of all strokes and results in high mortality, disability, and reduced quality of life [4]. The most common risk factors for SCI are atherosclerosis, hypertension, diabetes, and cardioembolism [5]. Iatrogenic SCI accounts for $45 \%$ of all SCI and can be caused by aortic surgery (0.3-6.5\%), epidural steroid injection, intra-aortic balloon pump, lumbar epidural catheter placement, or orthopaedic lumbar surgery $[6,7]$.

The spinal cord supply is divided into four semi-distinct territories: C1-T3 supplied by the vertebral arteries, T3-T7 supplied by the intercostal arteries, T8 to T12 supplied by the artery of Adamkiewicz, and the conus supplied by a medullary conus artery arising from the internal iliac artery $[4,7]$. A zone of less anastomoses is located between the $\mathrm{T} 4$ and $\mathrm{T} 8$ regions and may be susceptible to SCI [8].

In contrast to cerebral ischaemic infarction, where the guidelines for management and pre- and post-hospital care are well-established, there are no standard guidelines for the management of SCI, and there is no causative registered treatment except for acetylic acid. This is probably due to the low prevalence of SCI and its diverse aetiology and clinical presentation. In addition, to date there have been no large clinical trials evaluating the treatment options for SCI [9]. There are only some reports regarding SCI treatment with rt-PA, but the benefits of this therapy and its associated risks are unclear [10].

In response to the articles by Wyszomirski et al., Lattanzi et al. and Karaszewski et al., we would like to present the case of a patient with SCI treated off label with IV rt-PA who made a full recovery. A 74-year-old patient with a medical history of hypertension, coronary heart disease, heart infarction in 1992, and coronary artery bypass graft (CABG) in 2017, was admitted to our Neurology Department due to lower limb weakness which had started two hours before admission. The neurological examination revealed paraparesis without sensory deficit and sphincter disturbances (NIHSS $=6$, Rankin $=4$ ). The results of laboratory studies, cerebral computer tomography (CT) and CT-angiography, CT of the thoracic and lumbosacral spine, echocardiography and abdominal ultrasound all showed no abnormalities. Four hours after symptoms onset, the patient was treated with IV rt-PA, with neurological improvement in the form of diminished paraparesis. Cerebral CT performed after 24 hours was normal, but magnetic resonance imaging (MRI) of the spinal cord showed an infarct on the left side of the spinal cord extending from Th10 to Th11 segments. The patient was discharged from hospital with full recovery $(\mathrm{NIHSS}=0$, Rankin $=0)$.

So in relation to the articles written by Wyszomirski et al., Lattanzi et al. and Karaszewski et al., we would like to emphasise the urgent need to establish guidelines for management, pre- and post-hospital care in SCI. Up to now, only a few patients with SCI have been treated with IV rt-PA, but no randomised clinical trials have been registered. Our case report shows that SCI treatment with IV rt-PA can be very effective, and that randomised controlled trials or large observational studies are required to deliver more data. 


\section{References}

1. Wyszomirski A, Szczyrba S, Tomaka D, et al. Treatment of acute basilar artery occlusion: Systematic review and meta-analysis. Neurol Neurochir Pol. 2017; 51(6): 486-496, doi: 10.1016/i.pinns. 2017.07.012, indexed in Pubmed: 28823413.

2. Lattanzi S, Silvestrini M. Future challenges of stroke treatment. Neurol Neurochir Pol. 2018; 52(3): 415-416, doi: 10.1016/j. pjnns.2018.02.004, indexed in Pubmed: 29486885.

3. Karaszewski B, Wyszomirski A, Szczyrba S, et al. Response to the Letter "Future challenges of stroke treatment". Neurol Neurochir Pol. 2018; 52(3): 417-418, doi: 10.1016/i.pjnns.2018.02.006, indexed in Pubmed: 29685631.

4. Romi F, Naess H. Spinal Cord Infarction in Clinical Neurology: A Review of Characteristics and Long-Term Prognosis in Comparison to Cerebral Infarction. Eur Neurol. 2016; 76(3-4): 95-98, doi: 10.1159/000446700, indexed in Pubmed: 27487411.

5. Salvador de la Barrera S, Barca-Buyo A, Montoto-Marqués A, et al. Spinal cord infarction: prognosis and recovery in a series of 36 patients. Spinal Cord. 2001; 39(10): 520-525, doi: 10.1038/sj.sc.3101201, indexed in Pubmed: 11641795.
6. Robertson CE, Brown RD, Wijdicks EFM, et al. Recovery after spinal cord infarcts: long-term outcome in 115 patients. Neurology. 2012; 78(2): 114-121, doi: 10.1212/WNL.0b013e31823efc93, indexed in Pubmed: 22205760.

7. Novy J, Carruzzo A, Maeder P, et al. Spinal cord ischemia: clinical and imaging patterns, pathogenesis, and outcomes in 27 patients. Arch Neurol. 2006; 63(8): 1113-1120, doi: 10.1001/archneur.63.8.1113, indexed in Pubmed: 16908737.

8. Martirosyan NL, Feuerstein JS, Theodore N, et al. Blood supply and vascular reactivity of the spinal cord under normal and pathological conditions. J Neurosurg Spine. 2011; 15(3): 238-251, doi: 10.3171/2011.4.SPINE10543, indexed in Pubmed: 21663407.

9. Nasr DM, Rabinstein A. Spinal Cord Infarcts: Risk Factors, Management, and Prognosis. Curr Treat Options Neurol. 2017; 19(8): 28, doi: 10.1007/s11940-017-0464-3, indexed in Pubmed: 28688063.

10. Lee K, Strozyk D, Rahman C, et al. Acute spinal cord ischemia: treatment with intravenous and intra-arterial thrombolysis, hyperbaric oxygen and hypothermia. Cerebrovasc Dis. 2010; 29(1): 95-98, doi: 10.1159/000259618, indexed in Pubmed: 19923816. 